\title{
Influence of the density of states on the odd-even staggering in the charge distribution of the emitted fragments in nuclear heavy-ion collisions
}

\author{
N. L. Calleya, ${ }^{1}$ S. R. Souza, ${ }^{2,1}$ B. V. Carlson, ${ }^{3}$ R. Donangelo, ${ }^{2,4}$ W. G. Lynch, ${ }^{5}$ M. B. Tsang, ${ }^{5}$ and J. R. Winkelbauer ${ }^{5}$ \\ ${ }^{1}$ Instituto de Física, Universidade Federal do Rio Grande do Sul, Avenida Bento Gonçalves 9500, CP 15051, Porto Alegre 91501-970, Brazil \\ ${ }^{2}$ Instituto de Física, Universidade Federal do Rio de Janeiro Cidade Universitária, CP 68528, Rio de Janeiro 21941-972, Brazil \\ ${ }^{3}$ Departamento de Física, Instituto Tecnológico de Aeronáutica-CTA, São José dos Campos 12228-900, Brazil \\ ${ }^{4}$ Instituto de Física, Facultad de Ingeniería, Universidad de la República, Julio Herrera y Reissig 565, Montevideo 11300, Uruguay \\ ${ }^{5}$ National Superconducting Cyclotron Laboratory and Department of Physics and Astronomy Department, Michigan State University,
} East Lansing, Michigan 48824, USA

(Received 28 August 2014; revised manuscript received 6 October 2014; published 20 November 2014)

\begin{abstract}
The fragmentation of thermalized sources is studied using a version of the Statistical Multifragmentation Model which employs state densities that take the pairing gap in the nuclear levels into account. Attention is focused on the properties of the charge distributions observed in the breakup of the source. Since the microcanonical version of the model used in this study provides the primary fragment excitation energy distribution, one may correlate the reduction of the odd-even staggering in the charge distribution with the increasing occupation of high-energy states. Thus, in the framework of this model, such staggering tends to disappear as a function of the total excitation energy of the source, although the energy per particle may be small for large systems. We also find that, although the deexcitation of the primary fragments should, in principle, blur these odd-even effects as the fragments follow their decay chains, the consistent treatment of pairing may significantly enhance these staggering effects on the final yields. In the framework of this model, we find that odd-even effects in the charge distributions should be observed in the fragmentation of relatively light systems at very low excitation energies. Our results also suggest that the odd-even staggering may provide useful information on the nuclear state density.

DOI: 10.1103/PhysRevC.90.054616

PACS number(s): 25.70.Pq, 24.60.-k
\end{abstract}

\section{INTRODUCTION}

Size and isotopic correlations of fragments produced in nuclear reactions provide important information on both reaction mechanisms [1-4] and nuclear properties [1-8]. For instance, nuclear isoscaling $[9,10]$ turned out to hold information on the qualitative shape of the nuclear caloric curve [11], so that it may, in principle, provide a useful tool to help to establish experimentally the existence of the plateau predicted theoretically $[12,13]$ and observed in some experiments $[3,14,15]$. However, its properties have extensively been debated in the literature due to the uncertainties in determining both the temperature and the excitation energies $[7,16,17]$. The sensitivity of isoscaling to symmetry energy $[9,10,18,19]$ has stimulated investigations on the equation of state of the symmetry energy. However, further studies revealed that the relationship between symmetry energy and isoscaling is rather subtle [20-24], so that care must be taken in drawing conclusions based on such analyses.

Odd-even effects on the fragment charge distributions produced in different reactions have been recently reported in the literature [25-29]. Analyses based on the fragmentation of the quasiprojectile have been made at relativistic energies [25] as well as at much lower bombarding energies [28]. In both cases, clear odd-even effects have been observed in the fragment size distribution. This is surprising, to a certain extent, since the pairing gap should quickly vanish as the system is heated [30,31]. The data reported in Ref. [25] have been analyzed using an abrasion-evaporation model and the oddeven effects were attributed to the late stages of the evaporation process, during which the system is relatively cool. In Ref.
[28], it is demonstrated that the deexcitation of the primary hot fragments plays a very important role. Indeed, it was found that the adopted deexcitation process leads to the appearance of staggering effects in charge correlations of fragments with odd neutron excess, which is not observed in the primary fragments. Similar conclusions were also drawn in Ref. [26], where it was suggested that staggering should occur at low excitation energies. The study of central and semi-peripheral collisions carried out in Ref. [27] shows that important odd-even effects are observed for fragments with $Z<15$ and, in the case of peripheral collisions, they can be observed up to $Z=40$. On the other hand, other experimental results [29] reveal that these effects rapidly decrease as the fragment size increases.

In this work, we investigate the odd-even staggering using the version of the Statistical Multifragmentation Model (SMM) presented in Ref. [32]. In this implementation, the deexcitation of the primary fragments is treated by using a generalization of the Fermi breakup model (GFBM) [33], in which the emitted fragments are excited. As was demonstrated in Ref. [33], this is equivalent to the standard version of SMM if the same ingredients are employed in both treatments. It therefore allows one to investigate the role played by the pairing energy at different stages of the process if it is consistently taken into account in the model.

We thus start, in Sec. II, by reviewing the main features of the treatment presented in Ref. [32] and discussing the modifications of the model needed to include pairing effects in the nuclear state density. The predictions of the model are presented and discussed in Sec. III. Conclusions are drawn in Sec. IV. 


\section{THEORETICAL FRAMEWORK}

In the SMM [12,34,35] one assumes that an equilibrated source of mass and atomic numbers $A_{0}$ and $Z_{0}$, respectively, breaks up simultaneously after having expanded to a breakup volume $V=(1+\chi) V_{0}$, where $V_{0}$ is the volume corresponding to the normal nuclear density. We use $\chi=2$ in this work. Besides strict mass and charge conservation, each fragmentation mode must fulfill the energy conservation constraint

$$
E^{*}-B_{A_{0}, Z_{0}}=C_{c} \frac{Z_{0}^{2}}{A_{0}^{1 / 3}} \frac{1}{(1+\chi)^{1 / 3}}+\sum_{\{A, Z\}} n_{A, Z} E_{A, Z},
$$

where $E^{*}$ is the total excitation energy of the source and $B_{A_{0}, Z_{0}}$ denotes its binding energy. Except for fragments with $A \leqslant$ 4 , for which empirical values are adopted, we use the mass formula developed in Ref. [36]:

$$
B_{A, Z}=C_{v} A-C_{s} A^{2 / 3}-C_{c} \frac{Z^{2}}{A^{1 / 3}}+C_{d} \frac{Z^{2}}{A}+\delta_{A, Z} A^{-1 / 2},
$$

where

$$
C_{i}=a_{i}\left[1-k\left(\frac{A-2 Z}{A}\right)^{2}\right]
$$

and $i=v, s$ denotes the volume and surface terms, respectively. The last term in Eq. (2) is the usual pairing contribution to the binding energy:

$$
\delta_{A, Z}=\frac{1}{2}\left[(-1)^{A-Z}+(-1)^{Z}\right] C_{p} .
$$

The numerical values of all the parameters are listed in Ref. [36].

The Coulomb interaction between the fragments is taken into account in the framework of the Wigner-Seitz approximation [34,37]:

$$
\begin{aligned}
E_{\text {Coul }}= & C_{c} \frac{Z_{0}^{2}}{A_{0}^{1 / 3}} \frac{1}{(1+\chi)^{1 / 3}} \\
& +C_{c} \sum_{A, Z} n_{A, Z} \frac{Z^{2}}{A^{1 / 3}}\left[1-\frac{1}{(1+\chi)^{1 / 3}}\right],
\end{aligned}
$$

where $n_{A, Z}$ denotes the multiplicity of species $(A, Z)$. The contribution associated with the homogeneous sphere of volume $V$ is explicitly written on the right-hand side of Eq. (1), whereas the other terms are contained in $E_{A, Z}$, which reads

$$
E_{A, Z}=-B_{A, Z}+\epsilon_{A, Z}^{*}-C_{c} \frac{Z^{2}}{A^{1 / 3}} \frac{1}{(1+\chi)^{1 / 3}}+E_{A, Z}^{\text {trans }},
$$

where $\epsilon_{A, Z}^{*}$ represents the excitation energy of the fragment and $E_{A, Z}^{\text {trans }}$ is its translational energy.

In order to employ the efficient recursion formulas developed in Ref. [38], Eq. (1) is conveniently rewritten as

$$
Q \Delta_{Q} \equiv E^{*}-B_{A_{0}, Z_{0}}^{c}=\Delta_{Q} \sum_{\alpha, q_{\alpha}} q_{\alpha} n_{\alpha, q_{\alpha}}
$$

so that $Q$ is an integer number. In this way, the energy is discretized and the parameter $\Delta_{Q}$ controls the granularity of the discretization. The quantity $B_{A, Z}^{c}$ corresponds to

$$
B_{A, Z}^{c} \equiv B_{A, Z}+C_{c} \frac{Z^{2}}{A^{1 / 3}} \frac{1}{(1+\chi)^{1 / 3}}
$$

whereas

$$
q_{A, Z} \Delta_{Q} \equiv-B_{A, Z}^{c}+\epsilon_{A, Z}^{*}+E_{A, Z}^{\text {trans }} .
$$

The sum over $\alpha$ is carried out through all the isotopic species whereas that over $q_{\alpha}$ must be consistent with energy conservation, as stated in Eq. (7). Following Refs. [38] and [32], the average multiplicity of a species $(a, z)$, with energy $q \Delta_{Q}$, is given by

$$
\bar{n}_{a, z, q}=\frac{\omega_{a, z, q}}{\Omega_{A_{0}, Z_{0}, Q}} \Omega_{A_{0}-a, Z_{0}-z, Q-q} .
$$

The statistical weight $\Omega_{A, Z, q}$ is calculated through the following recurrence relation:

$$
\Omega_{A, Z, Q}=\sum_{\alpha, q_{\alpha}} \frac{a_{\alpha}}{A} \omega_{a_{\alpha}, z_{\alpha}, q_{\alpha}} \Omega_{A-a_{\alpha}, Z-z_{\alpha}, Q-q_{\alpha}}
$$

and $\omega_{A, Z, q}$ is obtained by folding the number of states associated with the kinetic motion with that corresponding to the internal degrees of freedom:

$$
w_{A, Z, q}=\gamma_{A} \int_{0}^{\epsilon_{A, Z, q}} d K \sqrt{K} \rho_{A, Z}\left(\epsilon_{A, Z, q}-K\right),
$$

where

$$
\gamma_{A}=\Delta_{Q} \frac{V_{f}\left(2 m_{n} A\right)^{3 / 2}}{4 \pi^{2} \hbar^{3}}
$$

$\epsilon_{A, Z, q} \equiv q \Delta_{Q}+B_{A, Z}^{c}, V_{f}=\chi V_{0}$ represents the free volume, $m_{n}$ is the nucleon mass, and $\rho_{A, Z}\left(\epsilon^{*}\right)$ is the density of the internal states of the nucleus $(A, Z)$ with excitation energy $\epsilon^{*}$. Thus, once the state density is specified, the above relations allow one to calculate the statistical properties of the system.

As mentioned above, except for the very light fragments, which have no internal degrees of freedom, the primary fragments are created in excited states, so that their deexcitation should be taken into account in order to obtain the final distribution. Different treatments have been adopted in the SMM $[2,39,40]$. In Ref. [40] an evaporation scheme based on the Weisskopf-Ewing treatment has been implemented. In other realizations of the model [2,39], it has been combined with the Fermi breakup model to describe the decay of light nuclei $(A \leqslant 16)$ while the Weisskopf-Ewing treatment is reserved for heavier fragments $(A>16)$. Recently, a generalization of the Fermi breakup model, including contributions due to the fragments' excited states, has been demonstrated to be formally equivalent to the SMM [33]. Its implementation to the deexcitation of the primary fragments was carried out in Ref. [32]. In this sense, our implementation of the fragments' deexcitation differs from the other two treatments just mentioned but the differences should not appreciably impact the qualitative properties of the charge distributions, on which we focus in this work.

Thus, the final fragment distribution is obtained by applying the above treatment successively for each fragment until they have decayed to the final states, as described in Ref. [32]. 
More specifically, each species $(A, Z)$ contributes to the yields of $(a, z)$ which add up to

$$
\bar{n}_{a, z, q} \rightarrow \bar{n}_{a, z, q}+\frac{\bar{n}_{a, z, q}^{\prime}}{1-\bar{n}_{A, Z, q_{0}}^{\prime}} \bar{n}_{A, Z, q_{0}}, \quad a<A .
$$

where

$$
\bar{n}_{a, z, q}^{\prime}=\frac{\omega_{a, z, q}}{\Omega_{A, Z, q_{0}}} \Omega_{A-a, Z-z, q_{0}-q} .
$$

Thus, by starting from the heaviest species down to the lightest fragment, one generates the final distribution.

The above relationships clearly show that the density of states plays a key role in the different stages of the process. The traditional SMM model employs [41]

$$
\rho_{A, Z}\left(\epsilon^{*}\right)=\rho_{\mathrm{SMM}}\left(\epsilon^{*}\right)=\rho_{\mathrm{FG}}\left(\epsilon^{*}\right) e^{-b_{\mathrm{SMM}}\left(a_{\mathrm{SMM}} \epsilon^{*}\right)^{3 / 2}}
$$

with

$$
\rho_{\mathrm{FG}}\left(\epsilon^{*}\right)=\frac{a_{\mathrm{SMM}}}{\sqrt{4 \pi}\left(a_{\mathrm{SMM}} \epsilon^{*}\right)^{3 / 4}} \exp \left(2 \sqrt{a_{\mathrm{SMM}} \epsilon^{*}}\right)
$$

and

$$
a_{\mathrm{SMM}}=\frac{A}{\epsilon_{0}}+\frac{5}{2} \beta_{0} \frac{A^{2 / 3}}{T_{c}^{2}}
$$

where $\epsilon_{0}=16.0 \mathrm{MeV}, \beta_{0}=18.0 \mathrm{MeV}$, and $T_{c}=18.0 \mathrm{MeV}$. The other parameters read $b_{\text {SMM }}=0.07 A^{-\tau}$ and $\tau=1.82(1+$ $A / 4500$ ), for $A>4$. In the case of the $\alpha$ particles, we set $\beta_{0}=0$ and $b_{\text {SMM }}=0.000848416$. For the other light nuclei with $A<5$, which have no internal degrees of freedom, we use $\rho_{A, Z}\left(\epsilon^{*}\right)=g_{A, Z} \delta\left(\epsilon^{*}\right)$, where $g_{A, Z}$ represents the empirical spin degeneracy factor. To avoid numerical instabilities at very small excitation energies, in this work, we use $\rho_{A, Z}\left(\epsilon^{*}\right)=$ $\rho_{0} e^{\left(\epsilon^{*}-U_{x}\right) / \tilde{\tau}}$ for $\epsilon^{*}<U_{x}$, where $U_{x}$ is defined below. The parameters $\rho_{0}$ and $\tilde{\tau}$ are adjusted, for each species, in order to match the value and the first derivative of the density of states at $\epsilon^{*}=U_{x}$.

Since this parametrization of the state density does not take pairing effects into account, this aspect is not consistently treated by the model. For this reason, these effects appear even at high excitation energies. This is illustrated in Fig. 1, which shows the charge distribution obtained in the breakup of the ${ }^{40} \mathrm{Ca}$ nucleus at different excitation energies. The primary and final yields are, respectively, displayed in Figs. 1(a) and 1(b). In both cases, odd-even staggering is clearly seen in the charge distribution, which, in the framework of the model, can be explained only by the presence of the pairing term in the fragments' binding energy [28], as is explicitly written in Eqs. (2) and (4). However, one should note that the breakup temperatures [see Eq. (23) below] vary from $T=3.8 \mathrm{MeV}$ for $E^{*} / A=1.5 \mathrm{MeV}$ to $T=5.0 \mathrm{MeV}$ for $E^{*} / A=3.5 \mathrm{MeV}$. In this temperature range, pairing effects should not be so important [30,31].

Empirical information on discrete states has been taken into account in the SMM version presented in Ref. [41]. However, extremely exotic nuclei enter in the above recursion formulas, for which information on such states is very scarce or unavailable. Furthermore, the quick growth of the number of states with the system size makes the implementation of this procedure extremely difficult, except in the case of very light

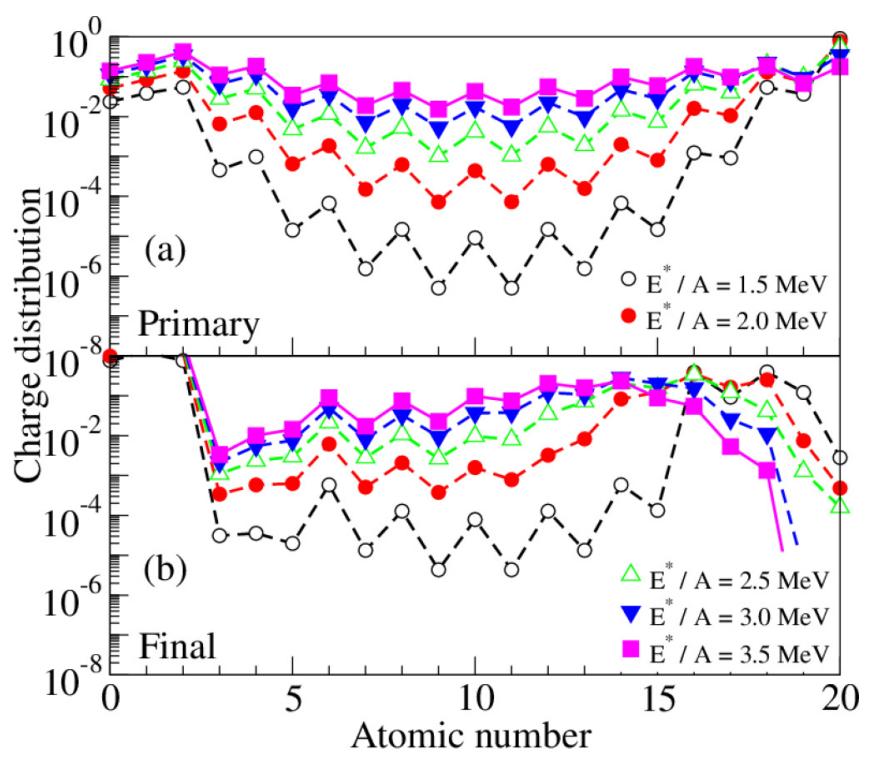

FIG. 1. (Color online) Charge distribution from the breakup of the ${ }^{40} \mathrm{Ca}$ nucleus at different excitation energies, showing primary (a) and final (b) yields, respectively. The standard SMM state density, Eq. (16), is used in all the cases. For details see the text.

nuclei. For this reason, in that work, analytical approximations have been used to supplement empirical information. There are different parametrizations of the nuclear level density in the literature, with different degrees of accuracy and complexity, such as those discussed in Refs. [31,42-45]. Since we intend to take into account the essential features of the nuclear level densities, we use the parametrization proposed by Gilbert and Cameron [42], as already employed in the SMM of Ref. [41]:

$$
\rho_{\mathrm{GC}}\left(\epsilon^{*}\right)= \begin{cases}\frac{\sqrt{2 \pi} \sigma_{0}}{\tau} e^{\left(\epsilon^{*}-E_{0}\right) / \tau}, & \epsilon^{*} \leqslant E_{x}, \\ \frac{\sqrt{\pi}}{12} \frac{e^{2 \sqrt{a\left(\epsilon^{*}-\Delta\right)}}}{\left(\epsilon^{*}-\Delta\right)\left(a\left[\epsilon^{*}-\Delta\right]\right)^{1 / 4}}, & \epsilon^{*}>E_{x},\end{cases}
$$

where $\Delta$ is the pairing energy of the nucleus, $E_{x}=U_{x}+\Delta$, $U_{x}=2.5+150 / A(\mathrm{MeV}), \sigma_{0}^{2}=0.0888 \sqrt{a\left(E_{x}-E_{0}\right)} A^{2 / 3}$, $E_{0}=E_{x}-\tau \log \left[\tau \rho_{2}\left(E_{x}\right)\right]$,

$$
\rho_{2}\left(E_{x}\right)=\frac{1}{12 \sqrt{2}} \frac{1}{\sigma_{0}} \frac{e^{2 \sqrt{a\left(E_{x}-\Delta\right)}}}{\left(E_{x}-\Delta\right)\left[a\left(E_{x}-\Delta\right)\right]^{1 / 4}}
$$

and

$$
\frac{1}{\tau}=\sqrt{\frac{a}{E_{x}-\Delta}}-\frac{3}{2} \frac{1}{E_{x}-\Delta} .
$$

For all nuclei, we use the level density parameter $a=A / 8.0 \mathrm{MeV}^{-1}$.

The low-energy part of this state density takes into account the fact that, in this energy domain, collective modes are also excited, besides those associated with single-particle states, so that the density of states is enhanced compared to that of a Fermi gas due to this extra contribution. For this reason, $\rho\left(\epsilon^{*}\right)$ increases as a function of $\exp \left(\epsilon^{*}\right)$ at low energies, instead of $\exp \left(2 \sqrt{a \epsilon^{*}}\right)$ as it does at higher energies. Its explicit dependence on $\epsilon^{*}-\Delta$ for $E>E_{x}$, rather than on $\epsilon^{*}$, takes into account the fact that the nucleon pairs must break in order 


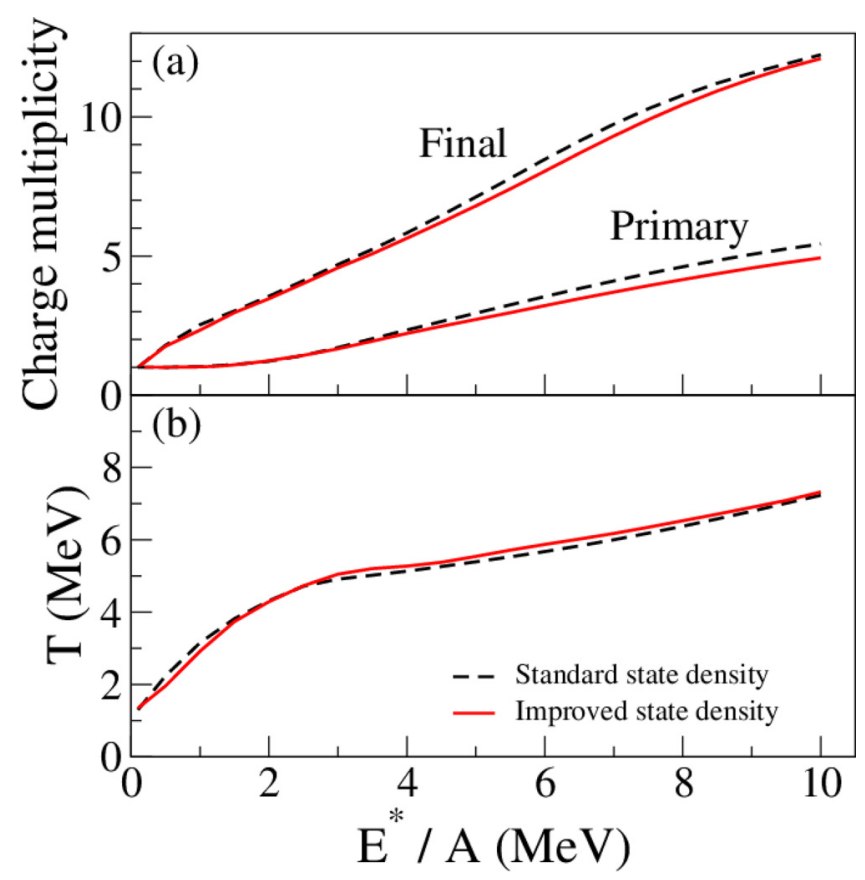

FIG. 2. (Color online) Comparison of different observables, from the breakup of the ${ }^{40} \mathrm{Ca}$ nucleus, obtained with the standard state density given by Eq. (16) and the improved formula, Eq. (22). For details see the text.

to excite single-particle states. In this way, the use of Eq. (19) takes into account pairing effects in the excited states.

Despite this desirable feature, this density of states differs from that adopted in the standard SMM, which should lead to predictions being at odds with previous results. Since we intend to preserve the properties of the model at high energies, we proceed as in Ref. [41] and gradually switch from $\rho_{\mathrm{GC}}$ to $\rho_{\mathrm{SMM}}$, so that we use

$$
\rho\left(\epsilon^{*}\right)=\rho_{\mathrm{GC}}\left(\epsilon^{*}\right)[1-f(x)]+\rho_{\mathrm{SMM}}\left(\epsilon^{*}-\Delta\right) f(x) .
$$

There is freedom in choosing the function $f(x)$, as long as it leads to a smooth switch from the two expressions for the density of states. We adopt $f(x)=[1+\tanh (x)] / 2$, with $x=\left[\epsilon^{*}-E_{x}-(1 / 2) \Delta E\right] / \delta E, \Delta E / A=\exp (-A / 35+1.2)$ $\mathrm{MeV}$, and $\delta E=10.0 \mathrm{MeV}$, which is simple and fulfills this requirement.

In order to check the extent to which the main properties of the model, particularly at high energies, are impacted by the replacement of Eq. (16) by Eq. (22), in Fig. 2(a) we display the primary and final charge multiplicities as a function of the excitation energy for the breakup of the ${ }^{40} \mathrm{Ca}$ nucleus, whereas Fig. 2(b) exhibits the corresponding caloric curve. The breakup temperature $T$ is calculated through

$$
\frac{1}{T}=\frac{\partial \ln \left(\Omega_{A_{0}, Z_{0}, Q}\right)}{\partial\left(Q \Delta_{Q}\right)} \approx \frac{\ln \left(\Omega_{A_{0}, Z_{0}, Q}\right)-\ln \left(\Omega_{A_{0}, Z_{0}, Q-1}\right)}{\Delta_{Q}} .
$$

The similarity of the results shown in Fig. 2 strongly suggests that this improved state density can be safely used, and so it is done from this point on.

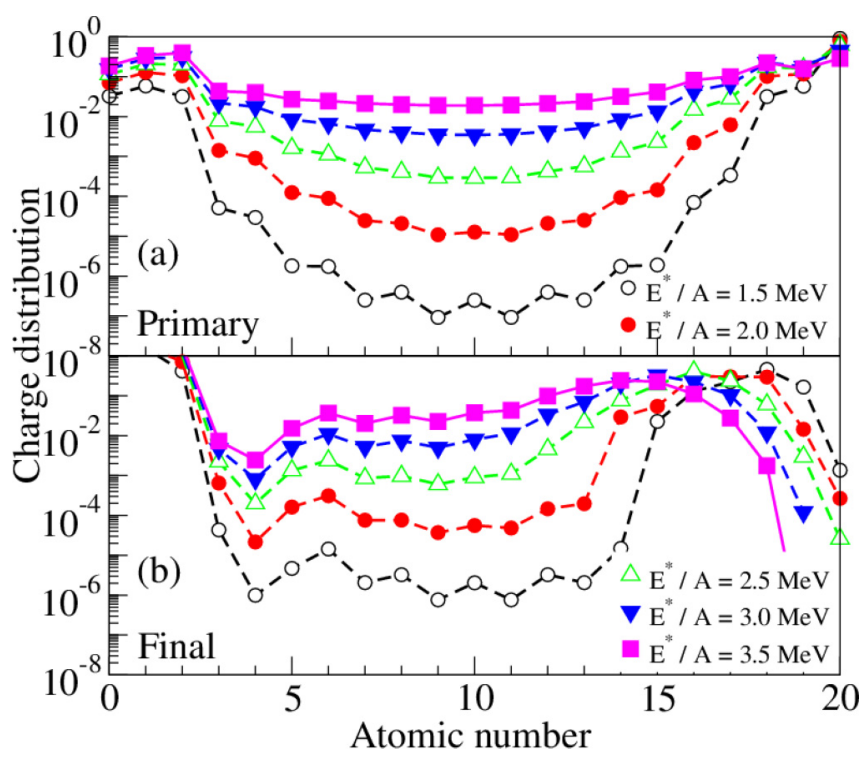

FIG. 3. (Color online) Same as Fig. 1 but the state density is replaced by Eq. (22). For details see the text.

\section{RESULTS}

We now investigate the role played by the improved state density given by Eq. (22) in the staggering properties of the charge distribution. In this way, the primary and final charge distributions for the fragmentation of the ${ }^{40} \mathrm{Ca}$ nucleus, at a few excitation energies, are displayed in Fig. 3. In contrast with the previous results shown in Fig. 1, odd-even effects in the primary distribution quickly disappear as the excitation energy increases, being barely noticeable at $E^{*} / A \approx 2.5 \mathrm{MeV}$. On the other hand, these effects are significantly enhanced in the final yields, as already suggested in former studies $[25,26,28]$. They also tend to be smoothed out as $Z$ increases, in agreement with the findings of Ref. [29].

In order to examine the dependence of the staggering on the source's size, we also consider the breakup of the ${ }^{80} \mathrm{Zr}$ nucleus. The corresponding primary and final charge distributions are exhibited in Fig. 4. The smoothing of the charge distribution is much more accentuated in this case than in the fragmentation of the ${ }^{40} \mathrm{Ca}$ nucleus. The magnitude of the staggering is very small at $E^{*} / A=1.5 \mathrm{MeV}$ and disappears almost completely at slightly higher excitation energies. The effects are somewhat more important at lower excitation energies, but the yields are extremely small.

The dependence of the odd-even staggering on the excitation energy, as well as on the fragment and system sizes, can be understood by examining the excitation energy distribution of the primary fragments. This is calculated through

$$
\bar{\epsilon}^{*}=\frac{\gamma_{a}}{\omega_{a, z, q}} \int_{0}^{\epsilon_{a, z, q}} d K\left(\epsilon_{a, z, q}-K\right) \sqrt{K} \rho\left(\epsilon_{a, z, q}-K\right) .
$$

The results are shown in Fig. 5, for the ${ }^{24} \mathrm{Mg},{ }^{28} \mathrm{Si}$, and ${ }^{32} \mathrm{~S}$ fragments, produced in the breakup of the ${ }^{40} \mathrm{Ca}$ and ${ }^{80} \mathrm{Zr}$ nuclei. Figure 5(a) displays the results corresponding to the ${ }^{40} \mathrm{Ca}$ source at $E^{*} / A=1.5 \mathrm{MeV}$. For each of the considered fragments, the vertical dotted lines represent $E_{x}$, which is the 


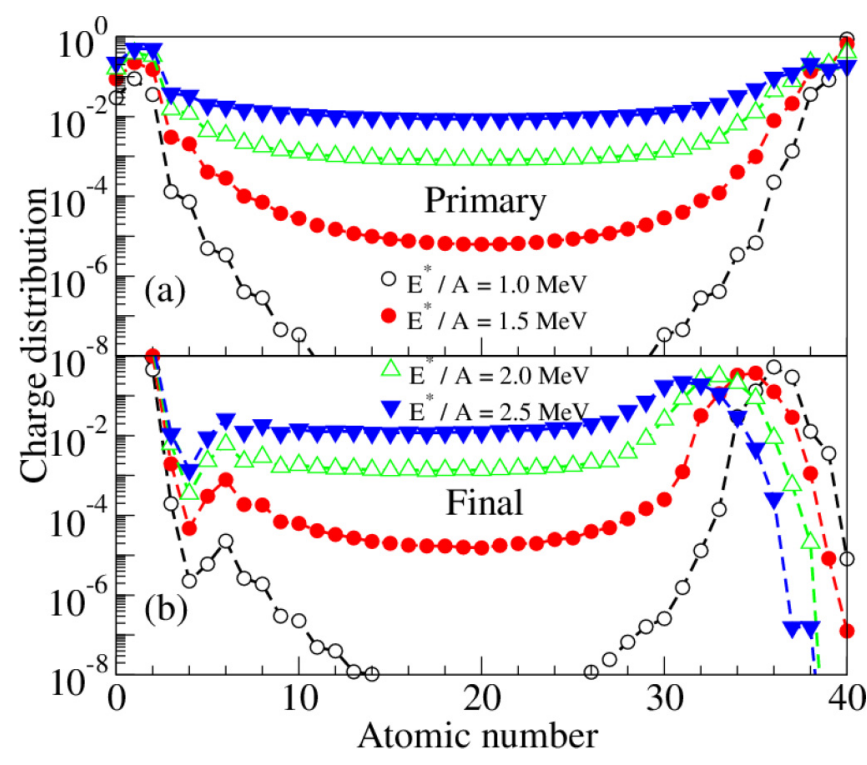

FIG. 4. (Color online) Same as Fig. 3 for the ${ }^{80} \mathrm{Zr}$ nucleus. For details see the text.

energy value below which collective states play a relevant role. One sees that the fraction of fragments produced with excitation energy higher than $E_{x}$ quickly increases with the fragment's size. Therefore, pairing effects should become progressively less important as the fragment's size increases.

Figure 5(b) shows that the excitation energy of the source also plays a very important role. Indeed, $E^{*} / A$ increases only by $0.5 \mathrm{MeV}$ from Fig. 5(a) to Fig. 5(b) but the fraction

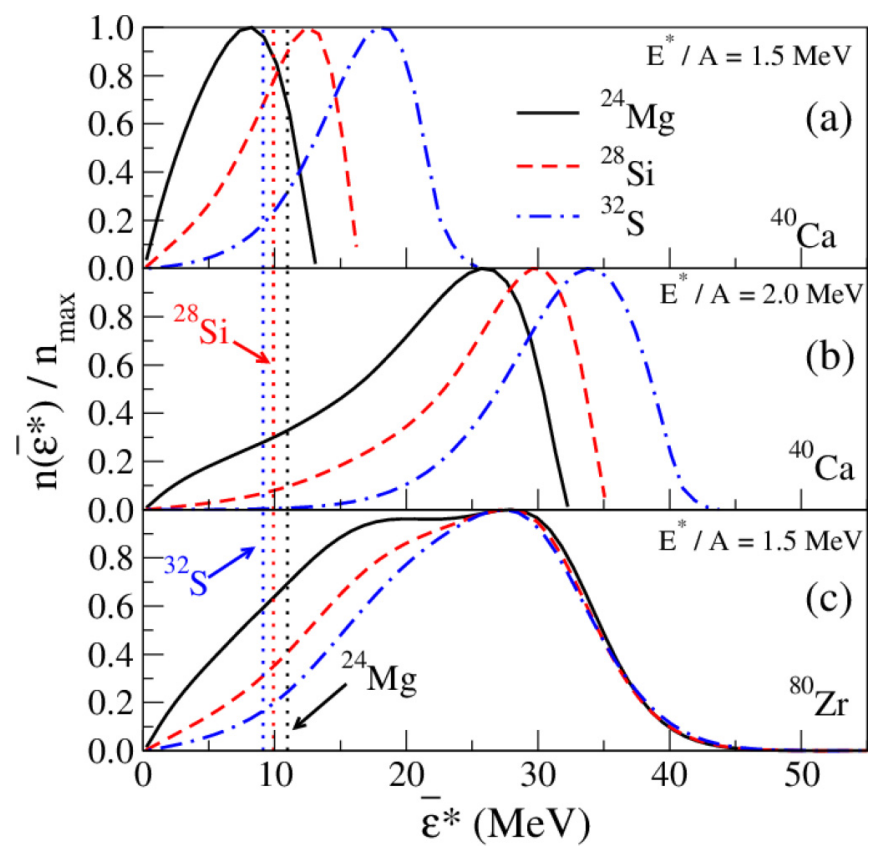

FIG. 5. (Color online) Average excitation energy of the primary fragments produced in the breakup of ${ }^{40} \mathrm{Ca}$ and ${ }^{80} \mathrm{Zr}$ nuclei. The vertical dotted lines represent $E_{x}$ for ${ }^{24} \mathrm{Mg},{ }^{28} \mathrm{Si}$, and ${ }^{32} \mathrm{~S}$, as indicated by the arrows and labels. For details see the text. of fragments with excitation energy below $E_{x}$ decreases substantially. This should significantly weaken the odd-even effects on the charge distribution, as is indeed noticed in Fig. 3.

In Fig. 5(c) is shown the average excitation energy for the same fragments, but for a ${ }^{80} \mathrm{Zr}$ nucleus as a source. The excitation energy per nucleon, $E^{*} / A=1.5 \mathrm{MeV}$, is the same as in Fig. 5(a), for the ${ }^{40} \mathrm{Ca}$ source. The much larger amount of the available excitation energy causes the energy distribution to become broader and its peak to move to higher excitation energies. Once more, the population with excitation energy below $E_{x}$ is significantly reduced, leading to smoother charge distributions. One should note that the breakup temperature is $T=3.89 \mathrm{MeV}$, which is very close to the value of $T=3.74 \mathrm{MeV}$ obtained with the ${ }^{40} \mathrm{Ca}$ source at the same excitation energy. These results reveal that, in this context, the total excitation energy, instead of the excitation energy per nucleon, is the relevant quantity.

Thus, in the framework of the SMM, the excitation energy, fragment's size, and source's size dependence are explained by the migration of the population in low-lying to high-lying states. Therefore, the study of odd-even staggering may help to obtain information on the nuclear state density.

Finally, we examine the extent to which it is possible to distinguish among different parametrizations of the pairing energy from this analysis. Besides that adopted in the SMM, whose amplitude is $\bar{\Delta} \equiv 11.86 / A^{1 / 2} \mathrm{MeV}$, we also carried out the calculations with the pairing term used in [46], in which case the amplitude reads $\bar{\Delta} \equiv 34.0 / A^{3 / 4}$. The comparison between these two terms is shown in Fig. 6(a), from which one sees that these prescriptions lead to important differences between the pairing energies. Notwithstanding this, the influence of this change of parametrization on the primary charge

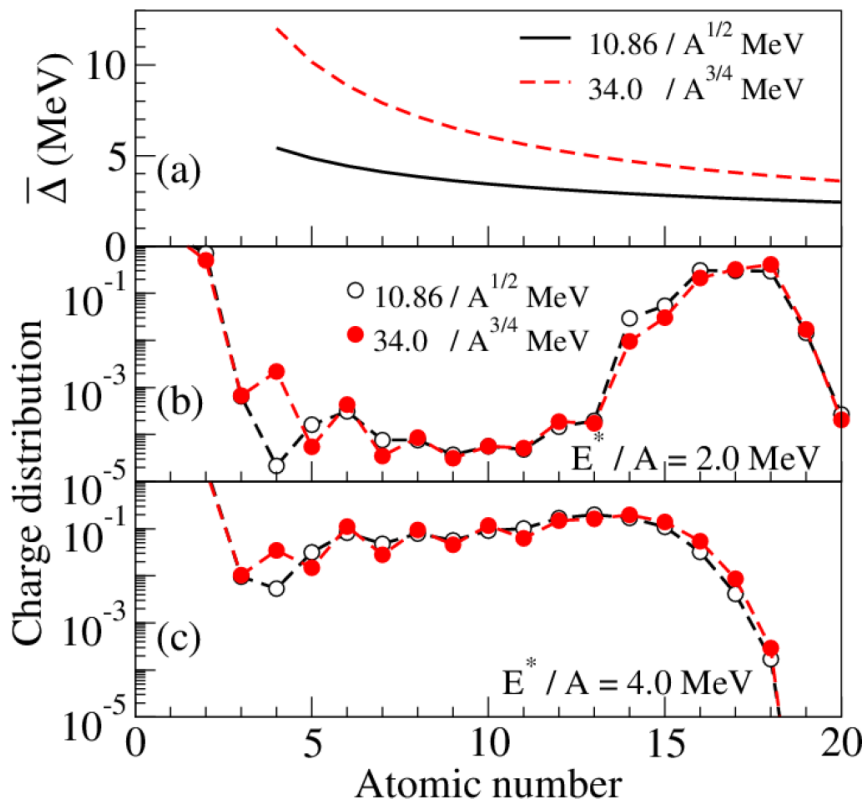

FIG. 6. (Color online) (a) Comparison between different pairing energies used in this work. (b) and (c) Comparison between the final charge distributions for the fragmentation of ${ }^{40} \mathrm{Ca}$ obtained with different symmetry energy parametrizations. For details see the text. 
distribution is very small, so they are not shown in this figure. The differences are amplified in the deexcitation process, as is illustrated in the middle and bottom panels of Fig. 6, which show the final charge distribution for the fragmentation of the ${ }^{40} \mathrm{Ca}$ nucleus at $E^{*} / A=2.0 \mathrm{MeV}$ and $E^{*} / A=4.0 \mathrm{MeV}$. The larger pairing energy clearly leads to more important odd-even effects and, therefore, the analyses made in Refs. [25-29] may be helpful in finding the best parametrization for the pairing term, but this requires a careful treatment of the deexcitation of the primary fragments in order to minimize ambiguities.

\section{CONCLUDING REMARKS}

By modifying the density of states employed in the SMM, in order to take the pairing energy into account, we have studied the odd-even staggering in the charge distribution of fragments produced in the breakup of excited nuclear systems. In agreement with previous results $[25,26,28]$, we find that this staggering is strongly influenced by the deexcitation of the primary hot fragments, so that it can be useful in tuning the treatments used to describe this stage of the process.

The smoothing of the charge distribution of primary fragments is explained in the framework of our model by the increasing of the population in states of energies for which the excitation of single-particle states becomes dominant in comparison to collective modes, being thus well described by a Fermi gas. Since the density of states is one of the ingredients of the calculations, our results suggest that the odd-even effects observed in the charge distribution may also be used to constrain this quantity.

We also found that a careful comparison with the experimental results may help to distinguish among different parametrizations of the pairing energy.

In conclusion, the sensitivity of this odd-even staggering to the key ingredients of the statistical calculations should be very useful in improving these models and constraining some of their parameters and assumptions. However, constraints brought in by angular momentum conservation, which is entirely disregarded in our model, may introduce correlations that can play a role in the staggering properties of the charge distribution. Therefore, deexcitation schemes based on the Hauser-Feshbach treatment, such as those discussed in Refs. [41,47], should also be employed.

\section{ACKNOWLEDGMENTS}

We would like to acknowledge CNPq, a FAPERJ BBP grant, FAPESP, and the joint PRONEX initiatives of CNPq/FAPERJ under Contract No. 26-111.443/2010 for partial financial support. This work was supported in part by the National Science Foundation under Grant No. PHY1102511. We also thank the Programa de Desarrollo de las Ciencias Básicas (PEDECIBA) and the Agencia Nacional de Investigación e Innovación (ANII) for partial financial support.
[1] L. G. Moretto and G. J. Wozniak, Annu. Rev. Nucl. Part. Sci. 43, 379 (1993).

[2] J. P. Bondorf, A. S. Botvina, A. S. Iljinov, I. N. Mihustin, and K. Sneppen, Phys. Rep. 257, 133 (1995).

[3] J. Pochodzalla, Prog. Part. Nucl. Phys. 39, 443 (1997).

[4] W. P. Tan et al., Phys. Rev. C 64, 051901 (2001).

[5] C. A. Ogilvie et al., Phys. Rev. Lett. 67, 1214 (1991).

[6] G. F. Peaslee et al., Phys. Rev. C 49, R2271 (1994).

[7] S. Das Gupta, A. Z. Mekjian, and M. B. Tsang, Adv. Nucl. Phys. 26, 89 (2001).

[8] C. B. Das, S. Das Gupta, W. G. Lynch, A. Z. Mekjian, and M. B. Tsang, Phys. Rep. 406, 1 (2005).

[9] H. S. Xu et al., Phys. Rev. Lett. 85, 716 (2000).

[10] M. B. Tsang, W. A. Friedman, C. K. Gelbke, W. G. Lynch, G. Verde, and H. S. Xu, Phys. Rev. Lett. 86, 5023 (2001).

[11] S. R. Souza, R. Donangelo, W. G. Lynch, W. P. Tan, and M. B. Tsang, Phys. Rev. C 69, 031607(R) (2004).

[12] J. P. Bondorf, R. Donangelo, I. N. Mishustin, and H. Schulz, Nucl. Phys. A 444, 460 (1985).

[13] D. Gross, Phys. Rep. 279, 119 (1997).

[14] J. Pochodzalla et al., Phys. Rev. Lett. 75, 1040 (1995).

[15] J. B. Natowitz, R. Wada, K. Hagel, T. Keutgen, M. Murray, A. Makeev, L. Qin, P. Smith, and C. Hamilton, Phys. Rev. C 65, 034618 (2002).

[16] E. Vient, Habilitation à diriger des recherches, Université de Caen, 2006, http://tel.archives-ouvertes.fr/tel-00141924

[17] B. Borderie and M. Rivet, Prog. Part. Nucl. Phys. 61, 551 (2008).

[18] M. B. Tsang et al., Phys. Rev. C 64, 054615 (2001).

[19] A. S. Botvina, O. V. Lozhkin, and W. Trautmann, Phys. Rev. C 65, 044610 (2002).
[20] S. R. Souza, M. B. Tsang, R. Donangelo, W. G. Lynch, and A. W. Steiner, Phys. Rev. C 78, 014605 (2008).

[21] S. R. Souza, M. B. Tsang, B. V. Carlson, R. Donangelo, W. G. Lynch, and A. W. Steiner, Phys. Rev. C 80, 041602(R) (2009).

[22] S. R. Souza, M. B. Tsang, B. V. Carlson, R. Donangelo, W. G. Lynch, and A. W. Steiner, Phys. Rev. C 80, 044606 (2009).

[23] G. Chaudhuri, F. Gulminelli, and S. Das Gupta, Phys. Rev. C 80, 054606 (2009).

[24] S. R. Souza and M. B. Tsang, Phys. Rev. C 85, 024603 (2012).

[25] M. Ricciardi, A. Ignatyuk, A. Kelić, P. Napolitani, F. Rejmund, K.-H. Schmidt, and O. Yordanov, Nucl. Phys. A 749, 122 (2005).

[26] M. D'Agostino et al., Nucl. Phys. A 861, 47 (2011).

[27] G. Casini et al., Phys. Rev. C 86, 011602 (2012).

[28] J. R. Winkelbauer, S. R. Souza, and M. B. Tsang, Phys. Rev. C 88, 044613 (2013).

[29] S. Piantelli et al. (FAZIA Collaboration), Phys. Rev. C 88, 064607 (2013).

[30] H. Kucharek, P. Ring, and P. Schuck, Z. Phys. A 334, 119 (1989).

[31] S. Goriely, Nucl. Phys. A 605, 28 (1996).

[32] S. R. Souza, B. V. Carlson, R. Donangelo, W. G. Lynch, and M. B. Tsang, Phys. Rev. C 88, 014607 (2013).

[33] B. V. Carlson, R. Donangelo, S. R. Souza, W. G. Lynch, A. W. Steiner, and M. B. Tsang, Nucl. Phys. A 876, 77 (2012).

[34] J. P. Bondorf, R. Donangelo, I. N. Mishustin, C. Pethick, H. Schulz, and K. Sneppen, Nucl. Phys. A 443, 321 (1985).

[35] K. Sneppen, Nucl. Phys. A 470, 213 (1987).

[36] S. R. Souza, P. Danielewicz, S. Das Gupta, R. Donangelo, W. A. Friedman, W. G. Lynch, W. P. Tan, and M. B. Tsang, Phys. Rev. C 67, 051602(R) (2003).

[37] E. Wigner and F. Seitz, Phys. Rev. 46, 509 (1934). 
[38] S. Pratt and S. Das Gupta, Phys. Rev. C 62, 044603 (2000).

[39] A. S. Botvina, A. S. Iljinov, I. N. Mishustin, J. P. Bondorf, R. Donangelo, and K. Sneppen, Nucl. Phys. A 475, 663 (1987).

[40] H. W. Barz, J. P. Bondorf, R. Donangelo, I. N. Mishustin, and H. Schulz, Nucl. Phys. A 448, 753 (1986).

[41] W. P. Tan, S. R. Souza, R. J. Charity, R. Donangelo, W. G. Lynch, and M. B. Tsang, Phys. Rev. C 68, 034609 (2003).

[42] A. Gilbert and A. G. W. Cameron, Can. J. Phys. 43, 1446 (1965).
[43] P. Demetriou and S. Goriely, Nucl. Phys. A 695, 95 (2001).

[44] T. von Egidy and D. Bucurescu, Phys. Rev. C 72, 044311 (2005).

[45] T. von Egidy and D. Bucurescu, Phys. Rev. C 73, 049901(E) (2006).

[46] P. Ring and P. Schuck, The Nuclear Many-body Problem (Springer-Verlag, New York, 1980).

[47] G. Baiocco, Ph.D. thesis, Universitá di Bologna and Université de Caen Basse-Normandie, 2012, http://amsdottorato. cib.unibo.it/4295/ 